\title{
COMPOSIÇÃO E EXTRAÇÃO DE NUTRIENTES POR TRACHY- POGON PLUMOSUS SOB ADUBAÇÃO E IDADES DE REBROTA
}

\author{
CHEMICAL COMPOSITION AND NUTRIENTS EXTRACTION OF \\ TRACHYPOGON PLUMOSUS UNDER FERTILIZATION AND REGROWTH AGES
}

\author{
Costa, N. de L. ${ }^{*}$; Moraes, A. ${ }^{2}$; Carvalho, P.C.F. ${ }^{3}$; Monteiro, A.L.G. ${ }^{4}$; Motta, A.C.V. ${ }^{5}$ \\ e Oliveira, R.A. ${ }^{2}$
}

\begin{abstract}
${ }^{1}$ Embrapa Roraima. Boa Vista, Roraima. Brasil. *newtonlucena@yahoo.com.br ${ }^{2}$ Departamento de Fitotecnia e Fitossanitarismo. UFPR. Curitiba, Paraná. Brasil. ${ }^{3}$ Departamento de Zootecnia. UFRGS. Porto Alegre, Rio Grande do Sul. Brasil. ${ }^{4}$ Departamento de Zootecnia. UFPR. Curitiba, Paraná. Brasil.

${ }^{5}$ Departamento de Solos. UFPR. Curitiba, Paraná. Brasil.
\end{abstract}

\section{Palavras chave adicionais}

Cálcio. Fibra em detergente ácido e neutro. Fósforo. Magnésio. Nitrogênio. Potássio.

\section{RESUMO}

No manejo de pastagens, a adequada disponibilidade de nutrientes do solo e a utilização de períodos de descanso compatíveis com as características morfofisiológicas da gramínea são fatores importantes para a obtenção de forragem de alta qualidade e, consequentemente, índices satisfatórios de produtividade animal. Avaliaramse os efeitos da correção da fertilidade do solo (testemunha, calagem, adubação e calagem + adubação) e da idade de rebrota (28, 42, 56, 70 e 84 dias) sobre a composição química e extração de nutrientes por Trachypogon plumosus nos cerrados de Roraima. Os teores de nitrogênio, fósforo, cálcio, magnésio e potássio foram inversamente proporcionais às idades de rebrota, ocorrendo o oposto quanto aos teores dos constituintes da parede celular. Os maiores teores de $\mathrm{N}$ e $\mathrm{K}$ foram obtidos com a adubação e a calagem + adubação, enquanto que a testemunha apresentou maiores teores de $\mathrm{P}, \mathrm{Ca}$ e $\mathrm{Mg}$. O efeito da idade de rebrota sobe a extração de nutrientes foi quadrático e os máximos valores registrados aos $67,8\left(26,71 \mathrm{~kg} \mathrm{ha}^{-1} \mathrm{de} \mathrm{N}\right), 63,4\left(2,82 \mathrm{~kg} \mathrm{ha}^{-1} \mathrm{de}\right.$ P), 62,9 (5,17 kg ha-1 de Ca), 68,4 (3,32 kg ha-1 de $\mathrm{Mg})$ e 66,0 dias $\left(12,71 \mathrm{~kg}^{-1} \mathrm{ha}^{-1}\right.$ de $\left.\mathrm{K}\right)$. A máxima extração de macronutrientes foi, em ordem decrescente, a seguinte: $\mathrm{N}>\mathrm{K}>\mathrm{Ca}>\mathrm{Mg}>\mathrm{P}$. A adubação e a calagem + adubação proporcionaram

\section{Additional KeYwords}

Acid detergent fiber. Calcium. Magnesium. Neutral detergent fiber. Nitrogen. Phosphorus. Potassium.

maior extração de nutrientes e forragem de melhor qualidade com menor teor de fibras.

\section{SUMMARY}

In pasture management, the availability of soil nutrients, and use of adequate rest periods, consistent with the grass morphological and physiological characteristics, are important factors for obtaining high quality forage and, consequently, satisfactory levels of animal production. To evaluate the effects of soil fertility correction (control, liming, fertilization and liming + fertilization) and regrowth ages $(28,42,56,70$ and 84 days) on chemical composition and nutrient extraction of Trachypogon plumosus in the Roraima's savannas. The nitrogen, phosphorus, calcium, magnesium and potassium contents were inversely proportional to regrowth ages, while cell wall components increased consistently with regrowth ages. The highest $\mathrm{N}$ and $\mathrm{K}$ contents were recorded with fertilization or liming + fertilization, while control or liming alone provided higher $\mathrm{P}, \mathrm{Ca}$ and $\mathrm{Mg}$ contents. Regrowth ages promoted quadratics effects on nutrients extraction and maximum values were estimated at $67.8\left(26.71 \mathrm{~kg} \mathrm{ha}^{-1}\right.$ of $\left.\mathrm{N}\right), 63.4$ (2.82 kg ha-1 of P), 62.9 (5.17 kg ha-1 of Ca), 68.4 (3.32 kg ha-1 de $\mathrm{Mg}$ ) and 66.0 days (12.71 $\mathrm{kg} \mathrm{ha}^{-1}$ 


\section{COSTA, MORAES, CARVALHO, MONTEIRO, MOTTA EOLIVEIRA}

of K). The nutrient extraction by the grass followed the decreasing order: $\mathrm{N}>\mathrm{K}>\mathrm{Ca}>\mathrm{Mg}>\mathrm{P}$. Fertilization and liming + fertilization improved nutrients extraction and forage quality with lower fiber contents.

\section{INTRODUÇÃO}

No processo de produção animal devese buscar produtos de alta qualidade e de baixo custo e com mínimo impacto sobre o ambiente, otimizando a utilização dos recursos naturais disponíveis e aumentando o retorno econômico e social em um sistema biologicamente sustentável (Soares et al., 2006). A produção de ruminantes em pastagens nativas é uma atividade ecologicamente sustentável, contudo, geralmente propicia, baixos índices produtivos que expõem a fragilidade econômica do sistema (Pinto et al., 2008). Em Roraima, os solos sob vegetação de cerrado abrangem uma área em torno de quatro milhões de hectares, caracterizados por baixa fertilidade natural (alta saturação de alumínio, baixa capacidade de troca catiônica e teores de fósforo extremamente baixos), onde ocorrem pastagens de gramíneas nativas, principalmente dos gêneros Andropogon, Paspalum, Axonopus, Aristida, Heteropogon e Trachypogon, as quais apresentam produtividade, valor nutritivo e capacidade de suporte baixos, o que limita o desempenho zootécnico da pecuária na região (Costa et al., 2012). Apesar de limitações quantitativas e qualitativas, historicamente, as pastagens nativas proporcionaram o suporte alimentar para a exploração pecuária, que ao longo dos anos, tornou-se a principal atividade econômica de Roraima (Costa et al., 2011). O pastoreio contínuo com taxa de lotação variável, mas em geral extensivo e desvinculado do ritmo estacional de crescimento das pastagens, tem contribuição direta para os baixos índices produtivos dos rebanhos (Mata et al., 1996; Sarmiento et al., 2006). O excesso de lotação animal e a não reposição dos nutrientes extraídos constituem as principais causas do esgotamento da fertilidade do solo do ecossistema e, consequentemente, da redução da capacidade de suporte e do potencial produtivo das pastagens nativas ao longo dos anos (Recio et al., 2011).

Nas áreas planas e não inundáveis dos cerrados de Roraima, Trachypogon plumosus é a gramínea predominante, constituindo entre 80 e $90 \%$ da composição botânica das pastagens nativas. A produtividade animal e a capacidade de suporte neste ecossistema são baixas, em decorrência da reduzida disponibilidade e qualidade da forragem, sendo necessário entre 6 e 10 ha de pastagem para a manutenção de cada bovino adulto, o que inviabiliza a economicidade da pecuária, desde que não sejam implementadas práticas para o seu melhoramento (Mata et al., 1996; Recio et al., 2011). A gramínea apresenta ciclo perene, hábito de crescimento cespitoso, plantas com 40 a 80 $\mathrm{cm}$ de altura e folhas pilosas. No entanto, são escassas as informações sobre o potencial produtivo e a resposta da gramínea à melhoria das condições do ambiente de produção, notadamente quanto à correção da fertilidade do solo, visando à proposição de práticas de manejo sustentáveis (Costa et al., 2012).

No manejo de pastagens busca-se o balanço harmônico entre crescimento da planta, eficiência de colheita da forragem e sua conversão em produtos de origem animal (Santos et al., 2008). A fertilidade do solo e a idade fisiológica da planta são os fatores que mais afetam a qualidade da forragem, representada pela interação entre sua composição química, digestibilidade, consumo voluntário e natureza dos produtos da digestão (Van Soest, 1994). Pastagens estabelecidas em solos de baixa fertilidade, na ausência de calagem e adubação, produzem forragem de baixo valor nutritivo, refletindo a incapacidade do solo em suprir satisfatoriamente a demanda metabólica da planta por nutrientes (Sarmiento et al., 2006; Pinto et al., 2008). A composição química das gramíneas forrageiras está estreitamente relacionada ao seu 
estádio de crescimento, como resultado das alterações morfológicas e fisiológicas que afetam o balanço entre a produção e a senescência de tecidos (Parsons et al., 2011). Com a maturidade da planta há redução na qualidade da forragem em decorrência dos altos teores de constituintes da parede celular (celulose, hemicelulose, lignina, pectina e sílica), reduzidos teores de proteína e minerais, além de elevada concentração de fibras indigestíveis (Parsons et al., 2011). Logo, deve-se procurar o ponto de equilíbrio entre produtividade e qualidade, visando assegurar os requerimentos nutricionais dos animais e garantindo, simultaneamente, a maximização da eficiência dos processos de produção, utilização e conversão da forragem produzida.

Neste trabalho foram avaliados os efeitos da correção e adubação do solo e da idade de rebrota sobre a composição química e a extração de nutrientes por Trachypogon plumosus nos cerrados de Roraima.

\section{MATERIALE MÉTODOS}

O experimento foi conduzido em uma pastagem nativa de T. plumosus, localizada em Boa Vista, Roraima $\left(60^{\circ} 43^{\prime}\right.$ de longitude oeste e $2^{\circ} 45^{\prime}$ de latitude norte), a qual não estava submetida a nenhuma prática de manejo. O clima da região, segundo a classificação de Köppen, é Awi, com precipitação anual de $1600 \mathrm{~mm}$, sendo que $80 \%$ ocorrem nos seis meses do período chuvoso (abril a setembro). O período experimental foi maio a agosto de 2011, que corresponde à estação chuvosa. Os dados de precipitação e temperatura foram coletados através de pluviômetro e termômetro instalados na área experimental (tabela I).

O solo da área experimental é um Latossolo Amarelo, textura média, com as seguintes características químicas, na profundidade de 0-20 cm: $\mathrm{pH}_{\mathrm{H} 2 \mathrm{O}}=5,1 ; \mathrm{P}=1,1 \mathrm{mg}$ $\mathrm{kg}^{-1}$ (Mehlich-1); $\mathrm{Ca}+\mathrm{Mg}=0,51 \mathrm{cmol}_{\mathrm{c}} \cdot \mathrm{dm}^{-3}$ (extração com acetato de cálcio $1 \mathrm{~N} \mathrm{pH}$ 7,0);
$\mathrm{K}=0,03 \mathrm{cmol}_{\mathrm{c}} \cdot \mathrm{dm}^{-3}$ (Mehlich-1); $\mathrm{Al}=0,39$ $\mathrm{cmol} \mathrm{dm}^{-3} ; \mathrm{H}+\mathrm{Al}=2,43 \mathrm{cmol} \cdot \mathrm{dm}^{-3}$ (extração com $\mathrm{KCl} 1 \mathrm{~N}) ; \mathrm{SB}=0,54 \mathrm{cmol} \cdot \mathrm{dm}^{-3} \mathrm{e} \mathrm{V}(\%)$ $=18,2$. O delineamento experimental foi em blocos ao acaso com três repetições e os tratamentos arranjados em um fatorial $4 \times 5$. Foram avaliados os efeitos de quatro estratégias de correção da fertilidade do solo (testemunha, calagem, adubação e calagem + adubação) e cinco idades de rebrota $(28,42,56,70$ e 84 dias após o corte de uniformização da pastagem a $5,0 \mathrm{~cm}$ acima da superfície do solo). O tamanho das parcelas foi de 5,0 x 4,0 m, sendo a área útil de $12 \mathrm{~m}^{2}$. A calagem foi realizada 30 dias antes do corte de uniformização da pastagem, visando elevar a $40 \%$ a saturação de bases $\left(650 \mathrm{~kg} \mathrm{ha}^{-1}\right.$ de calcário dolomítico $-\mathrm{PRNT}=$ $100 \%$ ). A adubação constou de $50 \mathrm{~kg} \mathrm{ha}^{-1} \mathrm{de}$ $\mathrm{N}$ (uréia), $50 \mathrm{~kg} \mathrm{ha}^{-1}$ de $\mathrm{P}_{2} \mathrm{O}_{5}$ (superfosfato triplo), $50 \mathrm{~kg} \mathrm{ha}^{-1}$ de $\mathrm{K}_{2} \mathrm{O}$ (cloreto de potássio) e $30 \mathrm{~kg} \mathrm{ha}^{-1} \mathrm{de} \mathrm{S}$ (enxofre elementar), aplicados a lanço após o rebaixamento da pastagem.

Os acúmulos de forragem, em cada idade de rebrota, foram estimados através de cortes mecânicos, realizados a uma altura de 5,0 $\mathrm{cm}$ acima da superfície do solo. O material colhido foi acondicionado em sacos de papel e pesado para estimativa da produção de biomassa aérea e, posteriormente, colocado

Tabela I. Precipitação (PP), temperaturas mínimas, máximas e médias e radiação solar $(R S)$ registradas durante o período experimental. Boa Vista, Roraima. 2011. (Rainfall (PP), minimum, maximum, and mean temperatures, and solar radiation (RS) recorded during the experimental period. Boa Vista, Roraima. 2011).

\begin{tabular}{|c|c|c|c|c|c|}
\hline \multirow{5}{*}{$\begin{array}{l}\text { Maio } \\
\text { Junho } \\
\text { Julho } \\
\text { Agosto }\end{array}$} & \multirow{2}{*}{$\begin{array}{c}\begin{array}{c}\mathrm{PP} \\
(\mathrm{mm})\end{array} \\
692,9\end{array}$} & \multicolumn{2}{|c|}{$\begin{array}{r}\text { Temperatura } \\
\text { mínima máxima }\end{array}$} & \multirow{2}{*}{$\begin{array}{l}\left({ }^{\circ} \mathrm{C}\right) \\
\text { média } \\
26,9\end{array}$} & \multirow{2}{*}{$\begin{array}{c}\begin{array}{c}\mathrm{RS} \\
\mathrm{MJ} / \mathrm{m}^{2}\end{array} \\
376,9\end{array}$} \\
\hline & & 23,0 & 30,8 & & \\
\hline & 383,8 & 22,7 & 31,7 & 27,2 & 381,8 \\
\hline & 389,2 & 22,2 & 32,1 & 27,1 & 432,4 \\
\hline & 234,8 & 23,8 & 32,9 & 28,4 & 458,8 \\
\hline
\end{tabular}


para secar em estufa de ventilação forçada a $65^{\circ} \mathrm{C}$ por 72 horas para determinação do acúmulo de matéria seca e submetido à moagem em peneira com malha de $5,0 \mathrm{~mm}$. Os teores de nitrogênio foram analisados de acordo com procedimentos descritos por Silva e Queiroz (2002), enquanto que os teores de $\mathrm{P}, \mathrm{Ca}, \mathrm{Mg}$ e $\mathrm{K}$ foram determinados conforme a metodologia descrita por Silva (1999). Os teores de Pe K foram quantificados após digestão nitroperclórica. O P foi determinado por colorimetria; o K por fotometria de chama e $\mathrm{Ca}$ e $\mathrm{Mg}$ por espectrofotometria de absorção atômica. Os teores de fibra em detergente neutro (FDN) e fibra em detergente ácido (FDA) foram determinados através da metodologia proposta por Van Soest et al. (1991). A extração dos nutrientes foi estimada multiplicando-se os teores de cada nutriente pela quantidade de forragem acumulada em cada idade de rebrota.

Os dados foram submetidos à análise de variância e de regressão considerando o nível de significância de $5 \%$ de probabilidade, utilizando-se o programa Sisvar (Ferreira, 2008). Para se estimar a resposta dos parâmetros avaliados às idades de rebrota, em função da correção e adubação do solo, a escolha dos modelos de regressão baseou-se na significância dos coeficientes linear e quadrático, por meio do teste $t$, de Student, ao nível de $5 \%$ de probabilidade.

\section{RESULTADOSEDISCUSSÃO}

Os efeitos da idade de rebrota sobre os teores dos macronutrientes presentes na biomassa aérea do $T$. plumosus foram lineares e negativos (figura 1). Resultado semelhante foi relatado por Souza Filho et al. (1999) para pastagens nativas dos cerrados do Amapá, cujos teores de N, P, Ca, Mg e $\mathrm{K}$ foram inversamente proporcionais ao período de rebrota (30, 60 e 90 dias). Com o desenvolvimento do dossel da pastagem o decréscimo na concentração de nutrientes, em função do maior período de rebrota, ocorre como consequência de dois fatores:
1) decréscimo na razão de área foliar (relação entre área foliar e a biomassa da planta), como consequêcncia de maior investimento dos nutrientes na produção de biomassa estrutural a qual não apresenta função fotossintetizante, visando promover o aumento em altura e tornar as plantas competitivas na captação da radiação solar incidente e 2) decréscimo na concentração de nutrientes por unidade de área das folhas sombreadas na porção inferior da planta, de modo a priorizar a alocação de nutrientes nas folhas superiores que apresentam maior taxa de fotossíntese (Parsons et al., 2011). No primeiro caso, a concentração de nutrientes na planta é uma função potencial negativa relacionada ao maior acúmulo de biomassa, notadamente durante o período vegetativo, sendo acentuada com baixa disponibilidade de nutrientes no solo, o qual não suprirá de forma satisfatória os requerimentos nutricionais da planta, evidenciando um efeito de diluição em decorrência do maior acúmulo de biomassa com o aumento da idade de rebrota. No segundo caso, quando há competição entre plantas, um dos mecanismos para aumentar a interceptação da radiação solar incidente é o aumento da estatura da planta, a qual deve investir nutrientes, notadamente o $\mathrm{N}$, para a produção de colmos, estruturas que apresentam baixa concentração de nutrientes (Parsons et al., 2011).

Este comportamento é bem descrito na literatura, podendo-se citar os resultados obtidos em Panicum repens por Tosati e Scheffer-Basso (2007) que observaram uma relação inversa entre teores de $\mathrm{N}$ e idades de rebrota $\left(1,58 ; 1,29 ; 1,28 \mathrm{e} 1,12 \mathrm{~g} \mathrm{~kg}^{-1}\right.$, respectivamente para $31,73,123$ e 152 dias). $\mathrm{Na}$ Venezuela, Cunha et al.(2003), em pastagens de $T$. plumosus, registraram teores de $\mathrm{N}$ de 1,$29 ; 1,14 ; 1,09$ e $0,91 \%$, respectivamente para plantas aos 15, 30, 45 e 60 dias de rebrota. Marcante et al. (2011) e Ribeiro e Pereira (2011) reportaram reduções superiores a $30 \%$ nos teores de N, P, Ca, Mg e K de Cynodon nlemfuensis x C. dactylon e 
Pennisetum americanum, respectivamente, ao comparem a composição química de plantas aos 28 e 56 dias de rebrota, enquanto que Mata et al. (1996), Crispim et al. (2003) e Stabile et al. (2010) constataram decréscimos de 67; 55 e $33 \%$ nos teores de $\mathrm{N}$ de $T$. plumosus, Andropogon bicornis e Panicum maximum 'Massai', respectivamente, com o aumento do período de rebrota de 30 para 60 dias.

Ao se considerar que teores de $\mathrm{N}$ inferiores a $11,2 \mathrm{~g} \mathrm{~kg}^{-1}$ de MS são limitantes para uma adequada fermentação ruminal, por implicarem em menor consumo voluntário, redução na digestibilidade da forragem e balanço nitrogenado negativo (Minson, 1984), observa-se na figura 1 A que a gramínea atenderia, satisfatoriamente, aos requerimentos mínimos dos ruminantes, em todos os períodos de rebrota, com o uso da adubação e calagem + adubação e até aos 70 dias para a testemunha e calagem.

Os teores de $\mathrm{Ca}$ e $\mathrm{Mg}$ (figuras 1C e 1D), independentemente da correção e adubação do solo e das idades de rebrota, foram superiores ao nível crítico para bovinos de corte
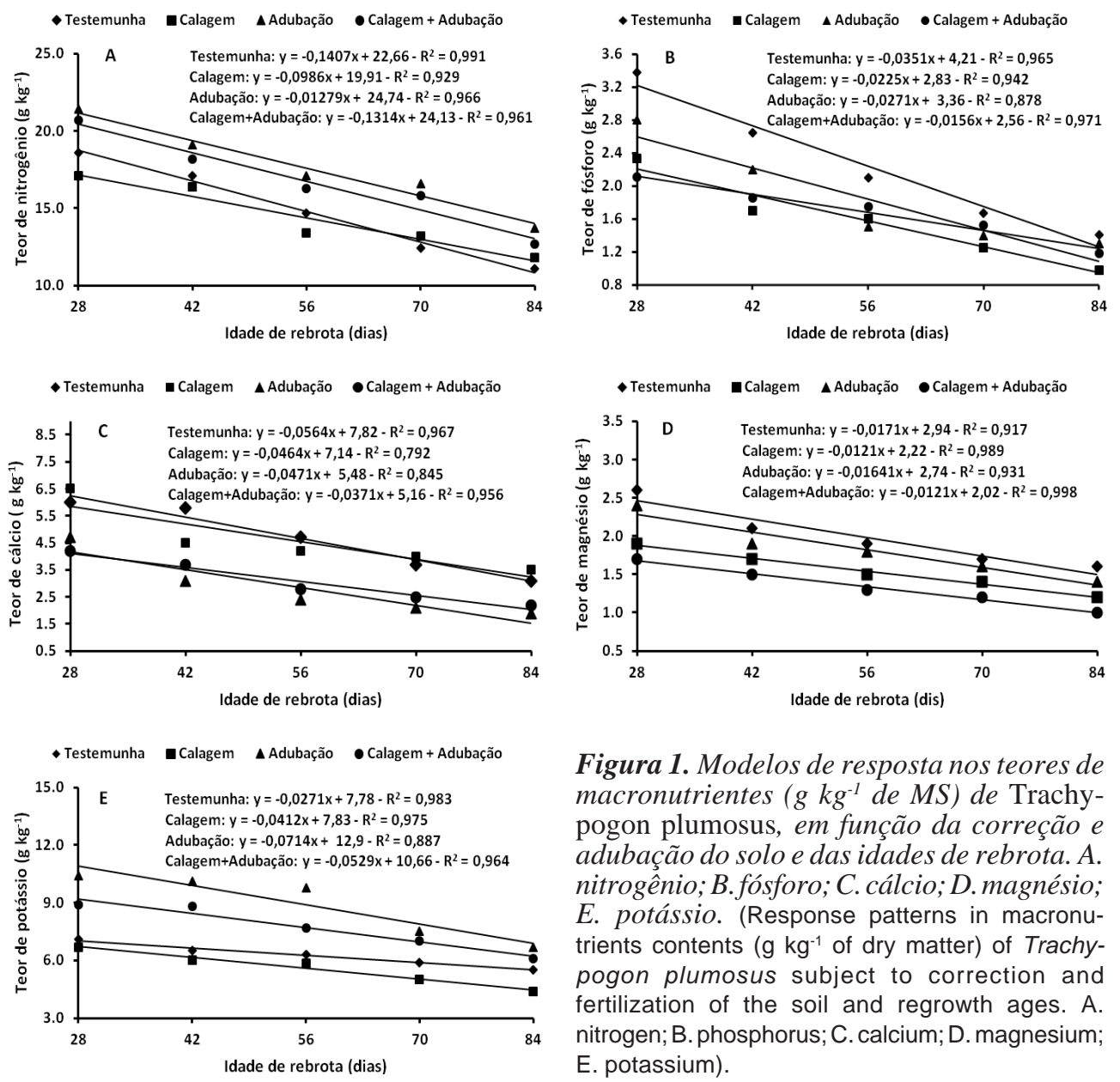

Figura 1. Modelos de resposta nos teores de macronutrientes ( $\mathrm{kg}^{-1}$ de MS) de Trachypogon plumosus, em função da correção $e$ adubação do solo e das idades de rebrota. A. nitrogênio; B. fósforo; C. cálcio; D. magnésio; $E$. potássio. (Response patterns in macronutrients contents $\left(\mathrm{g} \mathrm{kg}^{-1}\right.$ of dry matter) of Trachypogon plumosus subject to correction and fertilization of the soil and regrowth ages. A. nitrogen; B. phosphorus; C. calcium; D. magnesium; E. potassium).

Archivos de zootecnia vol. 62, núm. 238, p. 231. 


\section{COSTA, MORAES, CARVALHO, MONTEIRO, MOTTA E OLIVEIRA}

em crescimento $\left(1,8\right.$ e $1,0 \mathrm{~g} \mathrm{~kg}^{-1}$, respectivamente) estipulado pelo NRC (2000). Para o K $\left(6,5 \mathrm{~g} \mathrm{~kg}^{-1}\right)$ as exigências seriam atendidas com períodos de descanso de 42, 28, 84 e 70 dias, respectivamente para a testemunha, calagem, adubação e calagem + adubação, enquanto que para o $\mathrm{P}\left(1,8 \mathrm{~g} \mathrm{~kg}^{-1}\right)$ seus teores seriam adequados até 42 dias de rebrota, para todos os níveis de correção e/ ou adubação do solo (figuras 1E e 1B).

Independentemente da idade de rebrota, a composição química da parte aérea da gramínea, expressa em relação a MS, foi afetada $(\mathrm{p}<0,05)$ pela correção e adubação do solo (tabela II), apesar de sua boa adaptação aos solos ácidos e inférteis dos cerrados de Roraima, conforme constataram Costa et al. $(2011,2012)$. Os maiores teores de $\mathrm{N}\left(17,58 \mathrm{e} 16,74 \mathrm{~g} \mathrm{~kg}^{-1}\right)$ e de K $(8,90$ e 7,71 $\mathrm{g} \mathrm{kg}^{-1}$ ) foram obtidos com a utilização da adubação e calagem + adubação, respectivamente. Para o P $\left(2,24 \mathrm{~g} \mathrm{~kg}^{-1}\right), \mathrm{Ca}(4,66 \mathrm{~g}$ $\left.\mathrm{kg}^{-1}\right)$ e $\mathrm{Mg}\left(1,98 \mathrm{~g} \mathrm{~kg}^{-1}\right)$ as maiores concentrações foram registradas na testemunha, possivelmente como consequência de um efeito de concentração, em função de seus baixos rendimentos de forragem (tabela II). Estes valores foram superiores aos re-

Tabela II. Teores $\left(\mathrm{g} \mathrm{kg}^{-1}\right)$ de nitrogênio, fósforo, cálcio, magnésio e potássio de Trachypogon plumosus, em função dos níveis de correção e adubação do solo. Boa Vista, Roraima. 2011. (Nitrogen, phosphorus, calcium, magnesium and potassium contents $\left(\mathrm{g} \mathrm{kg}^{-1}\right)$ of Trachypogon plumosus, subject to correction and fertilization of the soil. Boa Vista, Roraima. 2011).

\begin{tabular}{lccccc}
\hline & $N$ & $P$ & $C a$ & $M g$ & $K$ \\
\hline Testemunha & $14,78^{\mathrm{b}}$ & $2,24^{\mathrm{a}}$ & $4,66^{\mathrm{a}}$ & $1,98^{\mathrm{a}}$ & $6,26^{\mathrm{b}}$ \\
Calagem(Cal) & $14,38^{\mathrm{b}}$ & $1,57^{\mathrm{d}}$ & $4,54^{\mathrm{b}}$ & $1,54^{\mathrm{c}}$ & $5,59^{\mathrm{b}}$ \\
Adubação (Ad) & $17,58^{\mathrm{a}}$ & $1,84^{\mathrm{bc}}$ & $2,84^{\mathrm{c}}$ & $1,82^{\mathrm{b}}$ & $8,90^{\mathrm{a}}$ \\
Cal-Ad & $16,74^{\mathrm{a}}$ & $1,68^{\mathrm{cd}}$ & $3,08^{\mathrm{d}}$ & $1,34^{\mathrm{d}}$ & $7,71^{\mathrm{a}}$ \\
\hline
\end{tabular}

abcdMédias seguidas de mesma letra nas colunas não diferem entre si pelo teste de Tukey ao níve de $5 \%$ de probabilidade.

Archivos de zootecnia vol. 62, núm. 238, p. 232. portados por Espinoza et al. (2002) para Trachypogon spp. $\left(11,3 ; 1,4 ; 3,2\right.$ e $5,5 \mathrm{~g} \mathrm{~kg}^{-1}$, respectivamente para $\mathrm{N}, \mathrm{P}, \mathrm{Ca}$ e $\mathrm{Mg}$ ); Wunsch et al. (2006) para pastagens nativas dos Campos de Cima da Serra no Rio Grande do Sul $\left(0,7 ; 4,1\right.$ e $1,0 \mathrm{~g} \mathrm{~kg}^{-1}$, respectivamente para $\mathrm{P}, \mathrm{Ca}$ e $\mathrm{Mg}$ ) e semelhantes aos estimados por Depablos et al. (2009) para $T$. plumosus $\left(2,1 ; 4,3 ; 1,8\right.$ e $8,2 \mathrm{~g} \mathrm{~kg}^{-1}$, respectivamente para $\mathrm{P}, \mathrm{Ca}, \mathrm{Mg}$ e $\mathrm{K}$ ). Em pastagens nativas das savanas africanas, com predominância de Panicum maximum, Ries e Shugart (2008) constataram efeitos significativos da adubação nitrogenada (200 $\mathrm{kg}$ de $\mathrm{N} \mathrm{ha}^{-1} \mathrm{ano}^{-1}$ ) e fosfatada (100 kg de $\mathrm{P}$ $\mathrm{ha}^{-1}$ ano $^{-1}$ ), isoladas ou combinadas, sobre os teores de $\mathrm{P}$ da gramínea, enquanto que os de $\mathrm{N}$ não foram afetados. Utilizando calagem e adubação semelhantes a do experimento, Barger et al. (2002), em pastagens de $T$. plumosus, relataram comportamento inverso, ou seja, efeito da adubação apenas sobre os teores de N. Na Venezuela, Rony et al. (1995) em pastagens de Dichanthium annulatum observaram que os teores de $\mathrm{N}$ e $\mathrm{P}$ foram diretamente proporcionais aos níveis de adubação nitrogenada e fosfatada. No entanto, D'Antonio e Mack (2006), em pastagens nativas de Melinis minutiflora, não detectaram variações significativas $(p>0,05)$ em seus teores de $\mathrm{N}$ e $\mathrm{P}$ com a aplicação isolada ou conjunta de $100 \mathrm{~kg}$ de $\mathrm{N}$ e de $\mathrm{P}_{2} \mathrm{O}_{5}$ ha $^{-1}$. Tendência semelhante foi verificada por Rocha et al. (2000) para $C$. dactylon 'Coastcross', cujos teores de N, P, $\mathrm{Ca}, \mathrm{Mg}$ e $\mathrm{K}$ não foram afetados pela adubação nitrogenada $(0,100,200$ e $400 \mathrm{~kg}$ de $\mathrm{N} \mathrm{ha}^{-1}$ ).

A relação entre idades de rebrota e a extração de macronutrientes na parte aérea da gramínea foi ajustada ao modelo quadrático de regressão (figura 2) e os máximos valores registrados com rebrotes de $68 ; 66 ; 63 ; 68$ e 63 dias sendo extraídos 26,$7 ; 12,7 ; 5,2 ; 3,3$ e 2,8 $\mathrm{kg} \mathrm{ha}^{-1} \mathrm{de} \mathrm{N}, \mathrm{K}, \mathrm{Ca}$, $\mathrm{Mg}$ e $\mathrm{P}$, respectivamente. Os decréscimos na absorção de nutrientes com o aumento da idade de rebrota decorrem da diminuição 
da capacidade fotossintética líquida do dossel, em função do sombreamento mútuo das folhas e da maior taxa respiratória das plantas, com reflexos diretos e negativos sobre as taxas de crescimento, implicando em menor demanda de nutrientes para o atendimento dos processos metabólicos da gramínea (Parsons et al., 2011). Em pastagens nativas dos cerrados do Amapá, Souza Filho et al. (1999) constataram maior absorção de nutrientes com períodos de descanso de 60 dias comparativamente a 90 dias $(7,68 ; 0,11$ e $0,13 \mathrm{~kg} \mathrm{ha}^{-1} v s .7,36 ; 0,10$ e $0,11 \mathrm{~kg} \mathrm{ha}^{-1}$, respectivamente para $\mathrm{N}, \mathrm{Ca}, \mathrm{e} \mathrm{Mg}$ ), valores
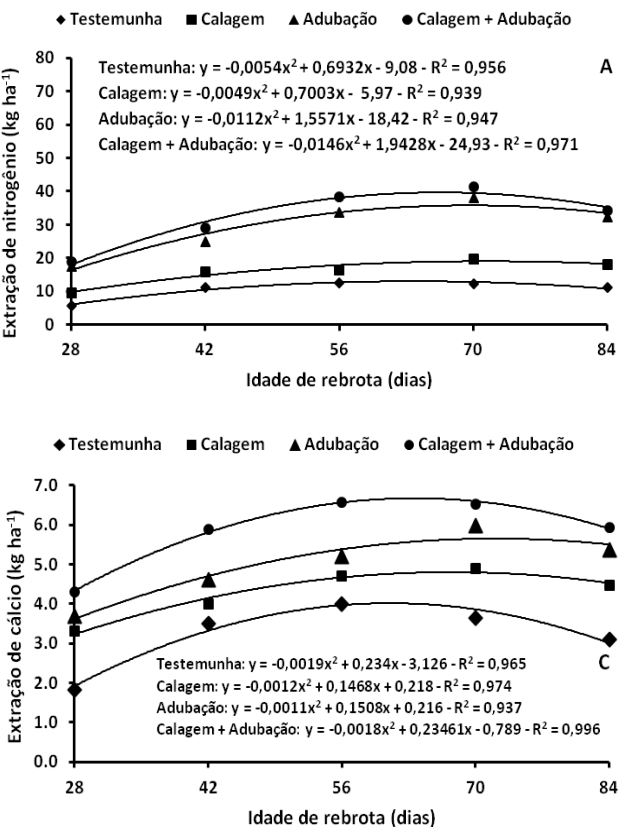

- Testemunha -Calagem \Adubação •Calagem + Adubação

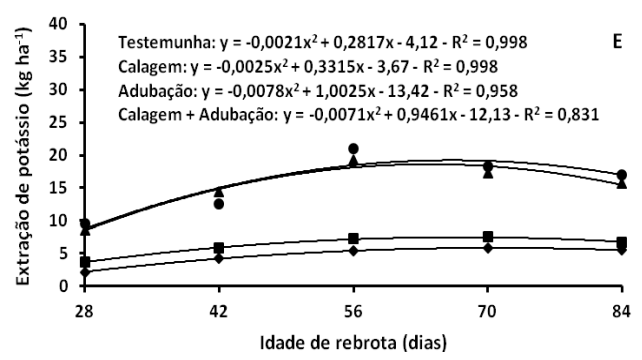

bem inferiores aos obtidos no presente experimento. Lemaire et al. (2008) observaram que a extração de $\mathrm{N}$ foi inversamente proporcional ao índice de área foliar e negativamente correlacionada com o acúmulo de forragem do Sorghum bicolor. Para todos os nutrientes, as quantidades extraídas foram inferiores às reportadas por Heringer e Jacques (2002) para pastagens nativas dos Campos de Cima da Serra no Rio Grande do Sul, sem queima ou roçagem $(41,3 ; 4,8$ e $4,1 \mathrm{~kg} \mathrm{ha}^{-1}$, respectivamente para $\mathrm{N}$, Ca e $\mathrm{Mg}$ ). Para assegurar produtividade primária líquida satisfatória de T. plumosus $(3500 \mathrm{~kg}$

- Testemunha - Calagem \Adubação • Calagem + Adubação

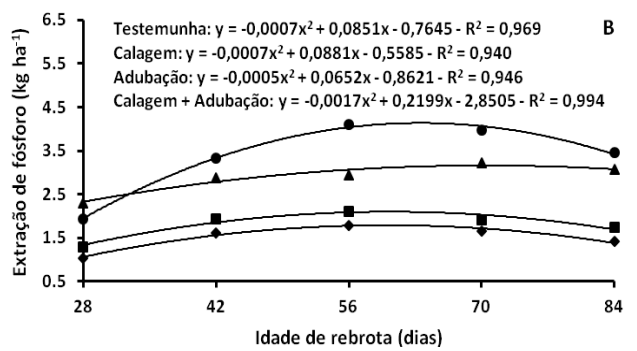

- Testemunha -Calagem $\triangle$ Adubação $\bullet$ Calagem + Adubação Testemunha: $y=-0,0005 x^{2}+0,0749 x-0,849 \cdot R^{2}=0,989$

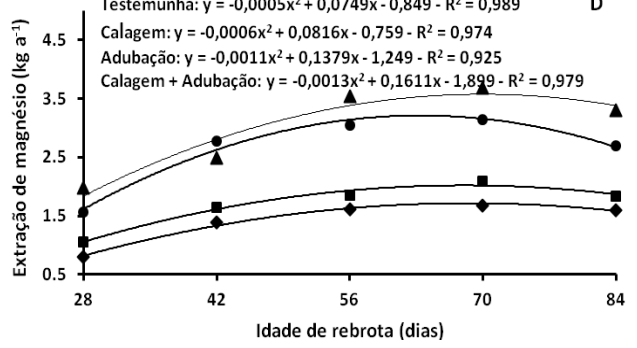

Figura 2. Modelos de resposta na extração de macronutrientes $\left(\mathrm{kg} \mathrm{ha}^{-1}\right)$ de Trachypogon plumosus, em função da correção e adubação do solo e das idades de rebrota. A. nitrogênio; B. fósforo; C. cálcio; D. magnésio; E. potássio. (Response patterns in macronutrients uptake $(\mathrm{kg}$ $\mathrm{ha}^{-1}$ ) of Trachypogon plumosus subject to correction and fertilization of the soil and regrowth ages. A. nitrogen; B. phosphorus; C. calcium; D. magnesium; E. potassium). 


\section{COSTA, MORAES, CARVALHO, MONTEIRO, MOTTA E OLIVEIRA}

de MS ha-1 ano $^{-1}$ ), Medina (1982) e Hernández et al. (2006) sugerem como adequadas extrações de $\mathrm{N}$ entre 30 e 43,6 kg de $\mathrm{N} \mathrm{ha}^{-1}$ $\mathrm{ano}^{-1}$. No presente experimento, as máximas extrações de $\mathrm{N}$ foram constatadas aos 69,5 $\left(35,7 \mathrm{~kg} \mathrm{ha}^{-1}\right)$ e 66,4 dias de rebrota $(39,5 \mathrm{~kg}$ $\left.\mathrm{ha}^{-1}\right)$, respectivamente para a adubação e calagem + adubação, as quais foram associadas a acúmulos de MS entre 2800 e $3200 \mathrm{~kg} \mathrm{ha}^{-1}$.

A máxima extração de macronutrientes pela parte aérea da gramínea, em ordem decrescente, foi a seguinte: $\mathrm{N}>\mathrm{K}>\mathrm{Ca}>\mathrm{Mg}$ $>\mathrm{P}$, independentemente da idade de rebrota. Resultados semelhantes foram obtidos por Costa et al. (2008) para Brachiaria brizantha 'Xaraés', enquanto que para $B$. brizantha 'Marandu', a absorção de K foi superior a de N (Costa et al., 2010). A extração desses nutrientes foi afetada $(\mathrm{p}<0,05)$ pela correção e adubação do solo (figura 2), as maiores quantidades absorvidas de $\mathrm{N}(25,47$ e 22,87 $\left.\mathrm{kg} \mathrm{ha}^{-1}\right), \mathrm{P}\left(2,67 \mathrm{e} 2,27 \mathrm{~kg} \mathrm{ha}^{-1}\right), \mathrm{Ca}(5,84 \mathrm{e} 4,97$ $\left.\mathrm{kg} \mathrm{ha}^{-1}\right), \operatorname{Mg}\left(2,64\right.$ e 2,99 $\left.\mathrm{kg} \mathrm{ha}^{-1}\right)$ e K $(15,67$ e $\left.15,05 \mathrm{~kg} \mathrm{ha}^{-1}\right)$ foram registradas com a utilização da calagem + adubação ou adubação isoladamente (tabela III). Da mesma forma, Barger et al. (2002) consta-

Tabela III. Extração $\left(\mathrm{kg} \mathrm{ha} \mathrm{a}^{-1}\right)$ de nitrogênio, fósforo, cálcio, magnésio e potássio de Trachypogon plumosus, em função dos níveis de correção ou adubação do solo. Boa Vista, Roraima. 2011. (Nitrogen, phosphorus, calcium, magnesium and potassium uptake $\left(\mathrm{kg} \mathrm{ha}^{-1}\right)$ by Trachypogon plumosus, subject to correction and fertilization of the soil. Boa Vista, Roraima. 2011).

\begin{tabular}{lccccc}
\hline & $\mathrm{N}$ & $\mathrm{P}$ & $\mathrm{Ca}$ & $\mathrm{Mg}$ & $\mathrm{K}$ \\
\hline Testemunha & $8,34^{\mathrm{c}}$ & $1,21^{\mathrm{b}}$ & $3,21^{\mathrm{d}}$ & $1,41^{\mathrm{b}}$ & $4,63^{\mathrm{b}}$ \\
Calagem (Cal) & $12,32^{\mathrm{b}}$ & $1,45^{\mathrm{b}}$ & $4,27^{\mathrm{c}}$ & $1,69^{\mathrm{b}}$ & $6,19^{\mathrm{b}}$ \\
Adubação (Ad) & $22,87^{\mathrm{a}}$ & $2,27^{\mathrm{a}}$ & $4,97^{\mathrm{b}}$ & $2,99^{\mathrm{a}}$ & $15,05^{\mathrm{a}}$ \\
Cal-Ad & $25,47^{\mathrm{a}}$ & $2,67^{\mathrm{a}}$ & $5,84^{\mathrm{a}}$ & $2,64^{\mathrm{a}}$ & $15,67^{\mathrm{a}}$ \\
\hline
\end{tabular}

abcdMédias seguidas de mesma letra nas colunas não diferem entre si pelo teste de Tukey ao nível de $5 \%$ de probabilidade. taram efeitos significativos da adubação nitrogenada (200 kg de $\left.\mathrm{N} \mathrm{ha}^{-1} \mathrm{ano}^{-1}\right)$ e fosfatada $\left(100 \mathrm{~kg} \mathrm{de}_{2} \mathrm{O}_{5}\right.$ ha $\left.^{-1} \mathrm{ano}^{-1}\right)$, isoladas ou combinadas, sobre a extração de $\mathrm{N}$ e $\mathrm{P}$ de T. plumosus. Para B. brizantha 'Marandu', Santos et al. (2008) obtiveram maior extração de $\mathrm{N}$ com a aplicação de $300 \mathrm{~kg}$ de $\mathrm{N} \mathrm{ha}^{-1}+$ $50 \mathrm{~kg}$ de $\mathrm{P}_{2} \mathrm{O}_{5}$ ha $^{-1}\left(1,83\right.$ g vaso $\left.^{-1}\right)$, comparativamente ao tratamento testemunha $(1,03 \mathrm{~g}$ vaso $\left.^{-1}\right)$ ou a aplicação isolada de $\mathrm{N}(1,39 \mathrm{~g}$ vaso $\left.^{-1}\right)$ ou $\mathrm{P}\left(1,31 \mathrm{~g} \mathrm{vaso}^{-1}\right)$. Para $B$. brizantha 'Marandu' e 'Xaraés', Costa et al. (2008, 2010) reportaram um efeito linear e positivo da aplicação de $\mathrm{N}$ e $\mathrm{P}$ sobre as extrações de $\mathrm{N}, \mathrm{P}, \mathrm{Ca}, \mathrm{Mg}$ e K.

Os teores de FDN e FDA foram diretamente proporcionais às idades de rebrota (figura 3). Resultados semelhantes foram relatados por Tosati e Scheffer-Basso (2007) para $P$. repens $(63,9 ; 68,1$ e $68,8 \%$ de FDN e 37,0; 39, 1 e 41,3\% de FDA, respectivamente para cortes aos 31,73 e 152 dias) e por Cunha et al. (2003) para T. plumosus $(26,6$; 28,$7 ; 35,5$ e $36,7 \%$ de FDA, respectivamente para 15, 30, 45 e 60 dias de rebrota). Em pastagens de $P$. maximum 'Mombaça', o aumento do período de rebrota de 30 para 90 dias, implicou em acréscimos de 10 e $32 \%$ nos teores de FDN (74,8vs. 82,4\%) e FDA (41,8vs. 55,3\%), respectivamente (Stabile et al., 2010). Para Paspalum dilatatum, Baréa et al. (2007) constataram que intervalos entre desfolhas de 30 dias $(67,3 \%$ de FDN e 44,9\% de FDA) proporcionaram forragem de melhor qualidade, comparativamente a intervalos de 45 dias $(71,8 \%$ de FDN e $46,8 \%$ de FDA). No entanto, Janusckiewicz et al. (2010) não detectaram efeito significativo do período de descanso ( 25 ou 35 dias) sobre os teores de FDN (74,36 e 73,13\%) e FDA $(46,27$ e $45,39 \%)$ de $P$. maximum 'Tanzânia'. Com o envelhecimento da planta a proporção dos componentes potencialmente digestíveis tende a diminuir e a de tecidos fibrosos, aumentar, sendo o conteúdo da parede celular o fator mais limitante ao desempenho produtivo de ruminantes consumindo gramíneas tropicais 

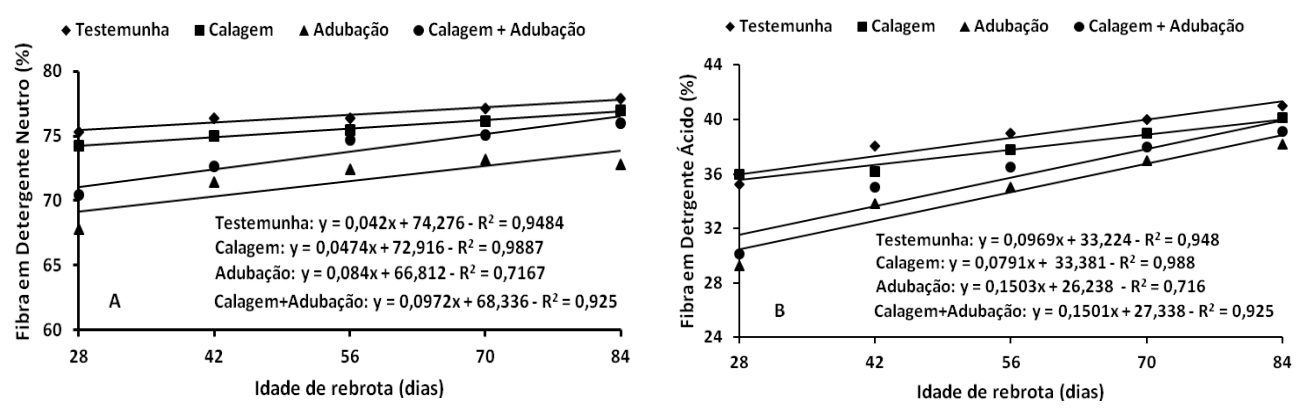

Figura 3. Teores (\% na MS) de fibra em detergente neutro $(A)$ e fibra em detergente ácido (B) de Trachypogon plumosus, em função da calagem e adubação e das idades de rebrota. (Contents (\%) of neutral fiber content (A) and acid fiber content (B) of Trachypogon plumosus, subject to correction and fertilization of the soil and regrowth ages).

(Castagnara etal., 2011). A FDN, constituída por celulose, hemicelulose, lignina e sílica, afeta diretamente o consumo voluntário, devido a maior taxa de enchimento e a menor de passagem do alimento no sistema digestivo, enquanto que a FDA se correlaciona negativamente com a disponibilidade de energia e a digestibilidade da forragem (Van Soest et al., 1991).

A correção e adubação do solo afetou $(p<0,05)$ os teores dos constituintes da parede celular da gramínea, independentemente da idade de rebrota, os quais foram reduzidos pela adubação (71,5\% de FDN de $34,6 \%$ de FDA) ou calagem + adubação (73,7 \% de FDN e $35,7 \%$ de FDA), ocorrendo o inverso com o uso apenas da calagem $(75,6 \%$ de FDN e $37,8 \%$ de FDA) e no tratamento testemunha $(76,6 \%$ de FDN e $38,6 \%$ de FDA) (tabela IV). Em gramíneas, a adubação ao estimular as taxas de aparecimento e alongamento de folhas, com reflexos positivos em seu comprimento final, contribui para redução ou manutenção dos teores de fibra, apesar dos acréscimos nos níveis de produtividade de forragem (Magalhães et al., 2011; Costa et al., 2012). Silva et al. (2012) reportaram efeitos positivos da adubação nitrogenada na redução dos teores de fibra de $P$. americanum $(55,4$; 53,$9 ; 53,5$ e $51,5 \%$ de FDN e 26,$3 ; 27,6 ; 26,0$ e $25,7 \%$ de FDA, respectivamente para 0,50 ,
100 e $150 \mathrm{~kg} \mathrm{de} \mathrm{Nha}^{-1}$ ), enquanto que Costa et al. (2006) e Santos et al. (2008), respectivamente em $B$. brizantha cv. MG-5 e $B$. decumbens cv. Basilisk, não constataram alterações significativas em seus teores de FDN e FDA, em função da adubação com N, $\mathrm{P}$ e K. Em pastagens de $P$. regnellii, Primavesi et al. (2008) reportaram tendência de elevação de seus teores de FDN como decorrência da aplicação de níveis crescentes de N, P e K.

Nos tratamentos com correção e aduba-

Tabela IV. Teores de fibra (\%) em detergente neutro (FDN) e fibra em detergente ácido (FDA) de Trachypogon plumosus, em função dos níveis de correção e adubação do solo. Boa Vista, Roraima. 2011. (Neutral detergent fiber and acid detergent fiber (\%) of Trachypogon plumosus, subject to correction and fertilization of the soil. Boa Vista, Roraima. 2011).

\begin{tabular}{lcc}
\hline & FDN & FDA \\
\hline Testemunha & $76,6^{\mathrm{a}}$ & $38,6^{\mathrm{a}}$ \\
Calagem & $75,5^{\mathrm{b}}$ & $37,8^{\mathrm{a}}$ \\
Adubação & $71,5^{\mathrm{d}}$ & $34,6^{\mathrm{b}}$ \\
Calagem + Adubação & $73,7^{\mathrm{c}}$ & $35,7^{\mathrm{b}}$ \\
\hline
\end{tabular}

abcdMédias seguidas de mesma letra nas colunas não diferem entre si pelo teste de Tukey ao nível de $5 \%$ de probabilidade. 


\section{COSTA, MORAES, CARVALHO, MONTEIRO, MOTTA E OLIVEIRA}

ção do solo, os teores de fibra na parte aérea da gramínea foram superiores aos limites sugeridos por Van Soest (1994), quais sejam 30 e $60 \%$ para FDA e FDN, respectivamente, como indicadores de forragem de alta qualidade, evidenciando os efeitos das altas temperaturas na formação de constituintes da parede celular, mecanismo utilizado por gramíneas tropicais para reduzir a herbivoria em decorrência da menor palatabilidade da forragem. Contudo, os teores foram inferiores aos relatados por Crispim et al. (2003) para Axonopus purpusii (78,1 \% de FDN e 45,5 \% de FDA) e Mesosetum chaseae $(75,6 \%$ de FDN e 48, 1 $\%$ de FDA), gramíneas presentes em pastagens nativas do Pantanal; Stabile et al. (2010) para P. maximum 'Mombaça' (76,7 $\%$ de FDN e 41,7 \% de FDA) e Pariz et al. (2011) para B. brizantha (78,7\% de FDN e $54,7 \%$ de FDA). Segundo Nussio et al. (1998), forragens com valores de FDA em

\section{BIBLIOGRAFIA}

Baréa, K.; Sheffer-Basso, S.M.; Dall'Agnol, M. e Oliveira, B.N. 2007. Manejo de Paspalum dilatatum Poir. biótipo Virasoro. 1. Produção, composição química e persistência. Rev Bras Zootecn, 36: 992-999.

Barger, N.N.; D'Antonio, C.M.; Ghneim, T.;Brink, K. and Cuevas, E.N. 2002. Nutrient limitation to primary productivity in a secondary savanna in Venezuela. Biotropica, 34: 493-501.

Castagnara, D.D.; Mesquita, E.E.; Neres, M.A.; Oliveira, P.S.R.; Deminicis, B.B. e Bamberg, R. 2011. Valor nutricional e características estruturais de gramíneas tropicais sob adubação nitrogenada. Arch Zootec, 60: 931-942.

Costa, K.A.P.; Araújo, J.L.; Faquin, V.; Oliveira, I.P.; Figueredo, F.C. e Gomes, K.W. 2008. Extração de macronutrientes pela fitomassa do capim-xaraés em função de doses de nitrogênio e potássio. Cienc Rural, 38: 1162-1166.

Costa, K.A.P.; Oliveira, I.P.; Faquin, V.; Machado, E.L.; Ramos, J.C. e Lima Filho, A.K. 2006. Efeitos quantitativo e qualitativa do nitrogênio e do potássio no desenvolvimento da Brachiaria torno de $40 \%$, ou mais, apresentam acentuada redução no consumo voluntário e na sua digestibilidade.

\section{CONCLUSÕES}

O aumento da idade de rebrota reduz linearmente a concentração e quadraticamente a extração de nutrientes da gramínea. As concentrações de cálcio e magnésio, independentemente da correção e adubação do solo e das idades de rebrota são adequados para suprir as exigências de bovinos de corte em crescimento, enquanto que para o nitrogênio, fósforo e potássio os requerimentos são atendidos com períodos de descanso de 70, 42 e 70 dias, respectivamente. A máxima extração de macronutrientes foi, em ordem decrescente, $\mathrm{N}>\mathrm{K}>$ $\mathrm{Ca}>\mathrm{Mg}>\mathrm{P}$. A adubação e a calagem + adubação proporciona maior extração de nutrientes e forragem de melhor qualidade com menor teor de fibras.

brizantha cv. MG-5. Rev Eletrônica Fac Montes Belos, 1: 56-70 (13/05/2012).

Costa, K.A.P.; Oliveira, I.P.; Severiano, E.C.; Sampaio, F.M.T.; Carijo, M.S. e Rodrigues, C.R. 2010. Extração de nutrientes pela fitomassa de cultivares de Brachiaria brizantha sob doses de nitrogênio. Cienc Anim Bras, 11: 307-314.

Costa, N. de L.; Gianluppi, V. e Moraes, A. 2012. Morfogênese de Trachypogon vestitus submetido à queima, nos cerrados de Roraima. Cienc Anim Bras, 13: 41-48.

Costa, N. de L.; Gianluppi, V.; Moraes, A. e Bendahan, A.B. 2011. Produtividade de forragem e características morfogênicas e estruturais de Axonopus aureus nos cerrados de Roraima. Amazônia Ciência \& Desenvolvimento, 6: 722.

Crispim, S.M.A.; Cardoso, E.L.; Rodrigues, C.A. e Barioni Júnior, W. 2003. Composição química da matéria seca de um campo de pastagem nativa submetido à queima, Pantanal, Mato Grosso do Sul, Brasil. ALPA, 11: 157-162.

Cunha, E.; Cabello, P. e Chicco, C.E. 2003. Compo- 


\section{ADUBAÇÃO DE TRACHYPOGON PLUMOSUS EM DIFERENTES IDADES DE REBROTA}

sición química y digestibilidad in vitro del Trachypogon sp. Agron Trop, 21: 183-193.

D'Antonio, C.M. and Mack, M.C. 2006. Nutrient limitation in a fire-derived, nitrogen-rich Hawaiian grassland. Biotropica, 38: 458-467.

Depablos, L.; Godoy, S.; Chicco, C.E. e Ordoñez, J. 2009. Nutrición mineral en sistemas ganaderos de las sabanas centrales de Venezuela. Zootec Trop, 27: 25-37.

Espinoza, F.M.; Díaz, Y.; Perdomo, E. y León, L. 2002. Utilización del banco de energia como estratégia de manejo en sabanas del estado Cojedes. II. Producción de matéria seca y valor nutritivo. Zootec Trop, 20: 11-20.

Ferreira, D.F. 2008. SISVAR: um programa para análises e ensino de estatística. Rev Symposium, 6: 36-41.

Heringer, E. e Jacques, A.V.A. 2002. Qualidade da forragem de pastagem nativa sob distintas alternativas de manejo. Pesqui Agropecu Bras, 37: 399- 406.

Hernández, D.L.; Santaella, S. and Chacón, P. 2006. Contribution of nitrogen-fixing organisms to the $\mathrm{N}$ budget in Trachypogon savannas. Eur J Soil Biol, 42: 43-50.

Janusckiewicz, E.R.; Magalhães, M.A.; Ruggieri, A.C. e Reis, R.A. 2010. Massa de forragem, composição morfológica e química de capimTanzânia sob diferentes dias de descanso e alturas de resíduo pós-pastejo. Biosci J, 26: 161-172.

Lemaire, G.; Oosterom, E.V.; Jeuffroy, M.H.; Gastal, F. and Massgnam, A. 2008. Crop species present different qualitative types of response to $\mathrm{N}$ deficiency during their vegetative growth. Field Crop Res, 105: 253-265.

Magalhães, A.F.; Pires, A.J.V.; Carvalho, G.G.P.; Sousa, R.S.; Silva, F.F.; Bonomo, P.; Veloso, C.M.; Magalhães, D.M.A. e Pereira, J.M. 2011. Composição química e concentração de nutrientes do capim braquiária adubada com nitrogênio e fósforo. Rev Bras Saúd Prod Anim, 12: 893-907.

Marcante, N.C.; Camacho, M.A. e Paredes, F.P.J. 2011. Teores de nutrientes no milheto como cobertura de solo. Biosci J, 27: 196-204.

Mata, D.; Herrera, P. y Birbe, B. 1996. Sistemas de producción animal con bajos insumos para las sabanas de Trachypogon spp. Ecotropicos, 9: 83-100.
Medina, E. 1982. Nitrogen balance in Trachypogon grasslands of central Venezuela. Plant Soil, 67: 305-314.

Minson, D.J.1984. Effects of chemical and physical composition of herbage eaten upon intake. In: Hacker, J.B. (Ed.). Nutritional limits to animal production from pasture. CAB. Farnham Royal. pp. 167-182.

NRC. 2000. National Research Council. Nutrient requirements of beef cattle. $7^{\text {th }}$ ed. National Academy Press. Washington. 242 pp.

Nussio, L.G.; Manazano, R.P. e Pedreira, C.G.S. 1998. Valor alimentício em plantas do gênero Cynodon. Simpósio sobre Manejo da Pastagem, 15. Piracicaba. Anais... FEALQ/ESALQ. Piracicaba. pp. 203-242.

Pariz, C.M.; Azenha, M.V.; Andreotti, M.; Araújo, F.C.M.; Ulian, N.A. e Bergamaschine, A.F. 2011. Produção e composição bromatológica de forrageiras em sistema de integração lavourapecuária em diferentes épocas de semeadura. Pesqui Agropecu Bras, 46: 1392-1400.

Parsons, A.; Rowarth, J.; Thornley, J. and Newton, P. 2011. Primary production of grasslands, herbage accumulation and use, and impacts of climate change. In: Lemaire, G.; Hodgson, J. e Chabbi, A. (Eds.). Grassland productivity and ecosystem services. CABI. Wallingford. pp. 318.

Pinto, C.E.; Fontoura Júnior, J.A.S.; Frizzo, A.; Freitas, T.M.S.; Nabinger, C. e Carvalho, P.C.F. 2008. Produções primária e secundária de uma pastagem natural da depressão central do Rio Grande do Sul submetida a diversas ofertas de fitomassa aérea total. Rev Bras Zootecn, 37: 1737-1741.

Primavesi, O.; Primavesi, A.C.; Batista, L.A. e Godoy, R. 2008. Adubação e produção de Paspalum em dois níveis de fertilidade de Latossolo Vermelho-Amarelo: estabelecimento e manutenção. Ciênc Agrotec, 32: 242-250.

Recio, L.P.; Lima, F.C. e Duran, A.O. 2011. Uso y manejo de forrajes nativos en la sabana inundable de la Orinoquia. Unillanos. Bogotá. 65 pp.

Ribeiro, K.G. e Pereira, O.G. 2011. Produtividade de matéria seca e composição mineral do capimTifton 85 sob diferentes doses de nitrogênio e idades de rebrotação. Ciênc Agrotec, 35: 811816.

Archivos de zootecnia vol. 62, núm. 238, p. 237. 


\section{COSTA, MORAES, CARVALHO, MONTEIRO, MOTTA E OLIVEIRA}

Ries, L.P. and Shugart, H.H. 2008. Nutrient limitations on understory grass productivity and carbon assimilation in a African woodland savanna. J Arid Environ, 72: 1423-1430.

Rocha, G.P.; Evangelista, A.R.; Paiva, P.C.A.; Freitas, R.T.F.; Garcia, E. e Rosa, B. 2000. Estudo da composição mineral de três gramíneas do gênero Cynodon. Ciênc Anim Bras, 1: 31-37.

Rony, T.; Muñoz, A. y Sequera, R. 1995. Efecto de nitrógeno y fósforo sobre el rendimiento y valor nutritivo del pasto tejano (Dichanthium annulatum Stapf.) en sabanas bajas de Portuguesa. Venezuela. Zootec Trop, 3: 63-80.

Santos, L.C.; Bonomo, P.; Silva, C.C.F.; Pires, A.J.V.; Veloso, C.M. e Patês, N.M.S. 2008. Produção e composição química da Brachiaria brizantha e Brachiaria decumbens submetidas a diferentes adubações. Ciênc Anim Bras, 9: 856-866.

Sarmiento, G.; Silva, M.P.; Naranjo, M.E. and Pinillos, M. 2006. Nitrogen and phosphorus as limiting factors for growth and primary production in a flooded savanna in the Venezuelan Llanos. $J$ Trop Ecol, 22: 203-212.

Silva, F.C. 1999. Manual de análises químicas de solos, plantas e fertilizantes. Embrapa Comunicação para Transferência de Tecnologia. Brasília. 370 pp.

Silva, D.J. e Queiroz, A.C. 2002. Análise de alimentos: métodos químicos e biológicos. 3. ${ }^{a}$ ed. UFV. Bogotá. $235 \mathrm{pp}$.

Silva, T.C.; Perazzo, A.F.; Macedo, C.H.O.; Batista, E.D.; Pinho, R.A.; Bezerra, H.F. e Santos, E.M. 2012. Morfogênese e estrutura de Brachiaria decumbens em resposta ao corte e adubação nitrogenada. Arch Zootec, 61: 91-102.

Soares, A.B.; Mezzalira, J.C.; Bueno, E.A.C.; Zotti, C.F.; Tirelli, L.A.; Cassol, L.C.; Marceniuk, L.V.; Adami, P.F. e Sartor, L.R. 2006. Efeitos de diferentes intensidades de pastejo em pastagem nativa melhorada sobre o desempenho animal. Rev Bras Zootecn, 35: 75-83.

Souza Filho, A.P.; Dutra, S. e Serrão, E.A.S. 1999 Produtividade estacional e composição química de Brachiaria humidicola e pastagem nativa de campo cerrado do Amapá, Brasil. Past Trop, 14: 11-16.

Stabile, S.S.; Salazar, D.R.; Jank, L.; Rennó, F.P. e Silva, L.F.P. 2010. Características de produção e qualidade nutricional de genótipos de capimcolonião colhidos em três estádios de maturidade. Rev Bras Zootecn, 39: 1418-1428.

Tosati, F.C. e Scheffer-Basso, S.M. 2007. Aspectos morfofisiológicos e bromatológicos do capim-torpedo (Panicum repens L., Poaceae). Rev Biotemas, 20: 7-14.

Van Soest, P.J. 1994. Nutritional ecology of the ruminant. 2..$^{\text {a }}$ ed. Cornell University Press. Corvallis. $476 \mathrm{pp}$.

Van Soest, P.J.; Robertson, J.B. and Lewis, B.A. 1991. Methods for dietary fiber, and no starch polysaccharides in relation animal nutrition. $J$ Dairy Sci, 74: 3583-3587.

Wunsch, C.; Barcellos, J.O.J.; Prates, E.R.; Costa, E.C.; Montanholi, Y.R. e Brandão, F. 2006. Macrominerais para bovinos de corte em pastagens nativas dos Campos de Cima da Serra, RS. Ciênc Rural, 36: 1258-1264. 